\title{
A Model for Predicting Migration from IPV4 to IPV6 by 2027 in Nigeria
}

\author{
Emmanuel Ekwonwune Nwabueze ${ }^{1 *}$, Ejike Nwosu C.. ${ }^{2}$, Oliver Eberechi Osuagwu1 \\ ${ }^{1}$ Department of Computer Science, Imo State University, Owerri, Nigeria \\ ${ }^{2}$ Department of Computer Science, Gregory University, Uturu, Nigeria \\ Email: *ekwonwuneemmanuel@yahoo.com
}

How to cite this paper: Ekwonwune, E.N., Ejike Nwosu C. and Osuagwu, O.E. (2018) A Model for Predicting Migration from IPV4 to IPV6 by 2027 in Nigeria. Open Journal of Modelling and Simulation, 6, 45-57.

https://doi.org/10.4236/ojmsi.2018.63004

Received: March 4, 2018

Accepted: July 10, 2018

Published: July 13, 2018

Copyright $\odot 2018$ by authors and Scientific Research Publishing Inc. This work is licensed under the Creative Commons Attribution International License (CC BY 4.0).

http://creativecommons.org/licenses/by/4.0/

\begin{abstract}
The present internet version which was established and consolidated over internet protocol version 4 (IPV4) in 1981, and whose amount of public addresses available is insufficient to meet the demands explosion and current internet multimedia devices, services and application intensive environment has posed serious problems of incomplete web transactions. Stakeholders and communication industry in Nigeria are unwilling and feel reluctant to migrate to IPV6 because of inhibiting factors. This needs urgent redress to overcome the tractions that are responsible for apathy to migration from IPV4 to IPV6 launched in 1994 by the Internet Engineering Task Force (IETF). If nothing is done, sometime, internet may run out of space, ARIN [1]. Users may suffer disillusionment and frustration. The objective of this study therefore is to design a model for predicting migration from IPV4 to IPV6 in Nigeria by 2027 based on growth trend developed from statistical indices. The essence is to explore and analyze the factors that can encourage migration to IPV6 in the next 10 years and use those factors to forecast growth, so that IPV6 will receive boost in terms of growth and patronage. The study also aims at designing a predictive model that simulates the behaviour of the restrictive policies on migration to $1 \mathrm{PV} 6$ so as to ascertain the current impact on non-motivation and unwillingness to migrate to IPV6 in Nigeria. The motivation behind this study is to identify the inhibiting factors responsible for lack of motivation to migrate from IPV4 to IPV6 in Nigeria. The methodologies that were deployed in packaging the model include the statistical methodology, Structured Systems Analysis and Design Methodology (SSADM) and prototyping. The result is indeed functional software, programmed through Visual Basic. Net. (VB.Net) that can be used to simulate the behavioural impact of any government policy formulation for Telecommunication industry and stakeholders.
\end{abstract}

\section{Keywords}

Model, Predicting, Migration, IPV4, IPV6 Inhibiting Factors 


\section{Introduction}

This study is about designing a model for predicting migration from internet protocol version 4 (IPV4) to internet protocol version 6. (IPV6). This is an actual process to fine tune and forecast the future growth trend trajectory of transitioning from IPV4 to IPV6 in Nigeria. This is motivated to see if its improvements can lead to the actual setup of a working and functional IPV6 and at the same time peep into the future implementation and workability of IPV6 in Nigeria. This will serve as a guide to policy formulation and a telecommunication gateway project for next generation internet diffusion in Nigeria. Implementing IPV6 is one thing, its workability is another, though it has been adopted skeletally by four (4) Internet Service Providers namely: Main One, Internet Solution, IPNX Nigeria and precisely Nigeria Research and Education Network, out of Thirty-five (35) organizations in Nigeria according to Olawale Ige, [2] an engineer and a former president, Nigeria Internet Group (NIG) and this has shown that migration from IPV4 to IPV6 in Nigeria is not being fully embraced as expected.

This is the area the predictive model of the study is very necessary. The study has clearly shown that migration to IPV6 acceptability and workability are dependent on government ability to revisit the policies and inhibiting factors responsible for lack of motivation to migrate from IPV4 to IPV6 in Nigeria. To appreciate the current status of migration from IPV4 to IPV6 in Nigeria and how ready Nigeria is, it is important to have an understanding and in-depth study of the existing (IPV4) system so as to identify some loopholes and at the same time suggest ways forward. However, a reliance on IPV4 outdated Network designs and technologies are leading to problems that are increasingly coming to light in the public domain. Overly, complex networks make the deployment of new applications and service updates challenging, slow and often high risk and the cost of maintaining the networks takes up a significant proportion of IT budgets, combined with infrastructure and service failures that can take a considerable amount of time to identify and resolve, these issues are increasingly hitting internet users and stakeholders satisfaction, creating demand for compensation and attracting the undesirable attention of Regulators. In spite of all these problems, yet there is no concrete plan either by the government or stakeholders (Vendors, ISPS, Users) in Nigeria to migrate to IPV6, "Next Generation Internet". The situation is indeed a threat to the Nation and constitutes economic, social and political doom. As a result of the above points, this study therefore tries to explore the inhibiting factors responsible for apathy to migration to IPV6 in Nigeria. Inhibiting factors were identified and addressed for the need of the organizations to ensure that their internet can support the current demand and future demand is paramount, by evolving IPV6, a fabric network design that overcomes many of the inherent weaknesses found in (IPV4) legacy systems. 


\section{Literature Review}

Since the inception of IPV4 in 1981 and migration from IPV4 to IPV6 designed by Internet Engineering Task Force (IETF) in 1994 at Toronto gathering, there have been several threads of researches revolving round its adoption and enhancement. This section therefore embodies the ideas drawn from the previous researchers' intuitions about the migration from IPV4 to IPV6. It is important to understand that, the internet, an acronym for international network, is defined according to Okafor, as a tool used to describe the networking of thousands of smaller networks around the world in [3]. Osuagwu O.E., in his view on prediction, stated that, in United States (US) for example, for 20 years, they needed \$25 billion to migrate from IPV4 to IPV6 in [4]. In collaborating with the statement above, which way Nigeria? Therefore, this should serve as a lesson to the developing countries like Nigeria. Wong and Anada, in their publication, revealed the solution to be what is now being pegged as the "Next Generation Internet Technology" a phenomenon called IPV6 in [5]. According to Susan Crawford of the Internet Corporation of names and numbers, the most serious threat to the internet in the $21^{\text {st }}$ Century will be a massive virtual black out known as a "distributed denial of service" in [6]. Therefore, there should be need for a smart choice to IPV6. Federal Agencies stated that Agencies may face brain drain if they delay transition from IPV4 to IPV6 in [7]. ARIN Board of Trustees passed a resolution advising the internet technical community that migration to a new version of the internet protocol, IPV6 will be necessary to allow continued growth of the internet in [1]. ARIN [1] said, sometime, the internet may run out of space, expediting the migration to IPV6 is the solution to the impending crisis as a result of coming shortage of internet protocol address of IPV4.

\section{Statement of Problems}

Despite the fact that migration from IPV4 to IPV6 has been recommended since 1994, as the "Next Generation Internet". The reasons being that, IPV4 address space has almost exhausted, it has neither produced the desired effect nor provided the needed impact on the sector in Nigeria. The stakeholders (vendors/ ISPS/Users) and the Telecommunication industry are still unwilling or reluctant to migrate to IPV6 technology and still operate with the prevalent (IPV4) system that can no longer accommodate todays always on, multimedia devices, services and application intensive environment. In this prevalent system, the underlying infrastructure has not kept pace with innovation in frontline operational system, leading to increasing pressure being placed on already overstretched back end infrastructure. Hence, major problems to be addressed in this study are to find out why Nigerians have not fully migrated to IPV6 and identify the inhibiting factors that are responsible for lack of motivation to migrate from IPV4 to IPV6 in Nigeria and suggest what can be done to migrate to IPV6 in Nigeria. This study tends to find out if there is anything government can do to motivate stakeholders to migrate to IPV6 and finally, 
identify the possibility to design a predictive model that will predict the growth trend of migration to IPV6 in Nigeria. This will serve as a guide to policy formulation in Nigeria.

\section{Aim and Objectives of Study}

The aim is to design a model that predicts the future of migration to IPV6 in Nigeria in the next 10 years. The system will be able to achieve the following functionalities or objectives:

1) Explore and analyze the factors inhibiting migration from IPV4 to IPV6 in Nigeria.

2) Develop migration to IPV6 Mathematical predictive model, based on growth trend developed from statistical indices.

3) Use the model to guide for policy formulation and for migration to IPV6 growth Trajectory in Nigeria.

\section{Significance of Study}

This study is necessary to fine tune and predicts the future growth trajectory of migration to IPV6, to see if its motivation can lead to functional migration to IPV6 setup and implementation, and that can improve communication in $\mathrm{Ni}$ geria. This also will position Nigeria to catch up with other developing economics like China and Ghana, etc. and also since other countries of the world like U.S, Singapore, Japan, Malaysia. Etc, are trying to keep pace with ever growing technological innovations, for the "Next Generation Internet", Nigeria should not be left behind. This study will serve as a guide to policy formulation in Nigeria and this leads to a gateway project for ICT diffusion in Nigeria by enabling the Ministry of Communication Technology and government to motivate the stakeholders so that migration to IPV6 may become feasible and viable.

\section{Methodology}

In this study, due to the nature of this research, the study deploys the following methodologies:

1) The standard procedure called "The Structured Systems Analysis and Design Methodology (SSADM)" a thorough fact-finding technique which was adopted in finding out and analyzing the existing system, its modes of operation and the challenges inherent in it.

2) Mathematical/Statistical methodology used to extract field data for analysis and interpretation.

3) Hypothetical-deductive methodology: this is an example of mathematical/ statistical methodology using questionnaire approach in which source data are subjected to analysis after being collected.

4) The Visual Basic. Net (VB.Net) was used to write the codes.

5) Finally, prototyping was used in packaging the model. 


\section{Model Formulation}

In the course of this study, the following mathematical models are employed:

1) Mathematical Models: Mathematical models grow out of equations that determine how a system changes from one state to the next (differential equations) and /or how one variable depends on the value or state of other variables (State Equations) these can also be divided into either numerical models or analytical models. For example, in the course of this study, a mathematical model was formulated thus:

$$
Y=a+B_{1} X_{1}+B_{2} X_{2}+\cdots+B_{n} X_{n}, B_{o}
$$

$a=$ Constant, where $\hat{Y}$ is the predicted or expected value of the dependent variable, $X_{1}$ through $X_{p}$ are $P$ distinct independent or predictor variables, $b_{o}$ is the value of $Y$ when all of the independent variables $\left(X_{1}\right.$ through $X_{p}$ ) are equal to zero, and $b_{1}$ through $b_{p}$ are the estimated Regression Coefficients. Each Regression Coefficient represents the change in $Y$ relative to a one unit change in the respective independent variable.

2) Statistical Models: A statistical model describes how one or more random variables are related to one or more other variables. The model is statistical when the variables are not deterministically but stochastically related in this study, the multiple regression were utilized in analyzing the data since the variables are stochastically related.

3) Hypothetic-Deductive models: is a proposed description of scientific method. According to it, scientific inquiry proceeds by formulating a hypothesis in a form that could conceivably be falsified by a test on observable data. A test that could and does run contrary to predictions of the hypothesis is taken as a falsification of the hypothesis. A test that could but does not run contrary to the hypothesis corroborates the theory. It is then proposed to compare the explanatory value of competing hypothesis by testing how stringently they are corroborated by their predictions. This model is very helpful since the researchers needed to ascertain if the variables identified as inhibiting factors for migration to IPV6 actually have relationship with unwillingness or reluctant to migrate to IPV6 in Nigeria. To assess also the impact of migration process to IPV6 since its introduction, a hypothesis is equally required. Hence, the choice of the hypothetic-deductive model.

\section{Model Assumptions}

This specifies the equations to be deployed in coding the simulator/predictor. Here multiple regression using ordinary differential equation (ODE) is applied. The general purpose of multiple regression (the term was first used by Pearson, 1908) in [8], is to learn more about the relationship between several independent or predictor variables and a dependent or criterion variable using a general model: $Y=a+b_{1} x_{1}+b_{2} x_{2}+\cdots+b_{n} x_{n}$ where, $a=Y$ intercept; points $b_{1}, b_{2}, \cdots, b_{n}$ $=$ the slope of $x_{1}, x_{2}, \cdots, x_{n}$ respectively. Explaining the model in a more general term, given a data set. $\left(y_{i}, x_{i 1}, \cdots, x_{i p}\right)_{i=1}^{T_{i}}$ of $n$ statistical units, a linear regression 
model assumes that the relationship between the dependent variable $Y_{i}$ and the p-vector of repressors $x_{i}$ is linear. This relationship is modelled through a disturbance term or error variable $\varepsilon_{i}$ an unobserved random variable that adds noise to the linear relationship between the dependent variable and regressors. Thus the model takes the form

$$
y_{i}=\beta_{1} x_{i 1}+\cdots+\beta_{P} x_{i \mathrm{P}}+\varepsilon_{i}=X_{i}^{\mathrm{T}} \beta+\varepsilon_{i}, i=1, \cdots, n
$$

where ${ }^{\mathrm{T}}$ denotes the transpose, so that $X_{i}^{\mathrm{T}} \beta$ is the inner product between vectors $X_{i}$ and $\beta$. Often these $n$ equations are stacked together and written in vector form as

$$
y=x \beta+\varepsilon \text { or } Y=a+B X
$$

where:

$$
y=\left(\begin{array}{c}
y_{1} \\
y_{2} \\
\vdots \\
y_{n}
\end{array}\right), x=\left(\begin{array}{c}
X_{1}^{\mathrm{T}} \\
X_{2}^{\mathrm{T}} \\
\vdots \\
X_{n}^{\mathrm{T}}
\end{array}\right)=\left(\begin{array}{ccc}
x_{11} & \cdots & x_{1 p} \\
x_{21} & \cdots & x_{2 p} \\
\vdots & \ddots & \vdots \\
x_{n 1} & \cdots & x_{n p}
\end{array}\right), \beta=\left(\begin{array}{c}
\beta_{1} \\
\beta_{2} \\
\vdots \\
\beta_{p}
\end{array}\right), \varepsilon=\left(\begin{array}{c}
\varepsilon_{1} \\
\varepsilon_{2} \\
\vdots \\
\varepsilon_{n}
\end{array}\right)
$$

The ordinary least squares (OLS) method minimizes the sum of squared residuals, and leads to a closed-form expression for the estimated value of the unknown parameter $\beta$ :

$$
\hat{\beta}=\left(X^{\mathrm{T}} X\right)^{-1} X^{\mathrm{T}} y=\left(\sum x_{i} x_{i}^{\mathrm{T}}\right)^{-1}\left(\sum x_{i} y_{i}\right)
$$

The estimator is unbiased and consistent if the errors have finite variance and are uncorrelated with the regressors.

$$
E\left[x_{i} \varepsilon_{i}\right]=0
$$

OR

$$
\begin{gathered}
\hat{Y}=b_{0}+b_{1} x_{1}+b_{2} x_{2}+\cdots+b_{p} x_{p} \\
a+B_{1} X_{1}+B_{2} X_{2}+\cdots+B_{n} X_{n} \\
B_{0}=a=\text { Constant }
\end{gathered}
$$

where $\hat{Y}$ is the predicted or expected value of the dependent variable, $X_{1}$ through $x_{p}$ are $p$ distinct independent or predictor variable, $b_{0}$ is the value of $Y$ when all of the independent variables $\left(x_{1}\right.$ through $\left.x_{p}\right)$ are equal to zero, and $b_{1}$ through $b_{p}$ are the estimated regression coefficients.

Each regression coefficient represents the change in $Y$ relative to a one unit change in the respective independent variable.

In the multiple regression situations, $b_{1}$ for example, is the change in $Y$ relative to a one unit change in $X_{1}$, holding all other independent variables constant (i.e., when the remaining independent variables are held at the same value or are fixed).The migration to IPV6 inhibiting factors Identification Number $\left(M_{1}\right.$ $M_{9}$ ) will be used for simulation, coding and manipulated in the equation to observe changes (positive or negative) that may occur due to changes in their beta 
coefficients. Hence, in this design we intend:

1) To use indices from the inhibiting factors' statistical analysis to stimulate the trajectory of future growth of migration to IPV6 if the trend remains linear or altered by policy summersaults.

2) Maintain a stakeholder's database and complaints database, produce meaningful reports for management and policy making.

3) The model can help to assist the stakeholders and government functionaries to try "what if" assessment of new telecommunication policies before, during \& after implementation.

\section{Analysis}

We analyzed the data using three scenarios as shown in Tables 1-3, and Figures 2-4:

1) Scenario 1: Testing Data for Scenario 1 as shown in Figure 1 (graph/output): The Scenario 1 are the beta coefficients of the inhibiting factors as obtained from SPSS analysis as shown in Table 1: $M_{1}=0.181, M_{2}=0.175, M_{3}=0.186, M_{4}=$ $0.194, M_{5}=0.195, M_{6}=0.190, M_{7}=0.184, M_{8}=0.191, M_{9}=0.202$, Base year $=$ 2017, year Gap = ten (10) years.

The results are as shown in Table 1 and Figure 2

Scenario 1: Trajectory graph as shown in Figure 2:

2) Scenario 2: this scenario II tested the system, when the beta coefficients are removed or improved upon. The results are shown in Table 2. And Figure 3.

Testing data for scenario II:

$$
\begin{aligned}
& M_{1}=0.381, M_{2}=0.475, M_{3}=0.386, M_{4}=0.491, \\
& M_{5}=0.395, M_{6}=0.490, M_{7}=0.394, M_{8}=0.391 \\
& M_{9}=0.402: \text { Base year } 2017, \text { year Gap = ten }(10) \text { years. }
\end{aligned}
$$

Table 2 shows when the inhibiting factors are removed/improved or adjusted positively.

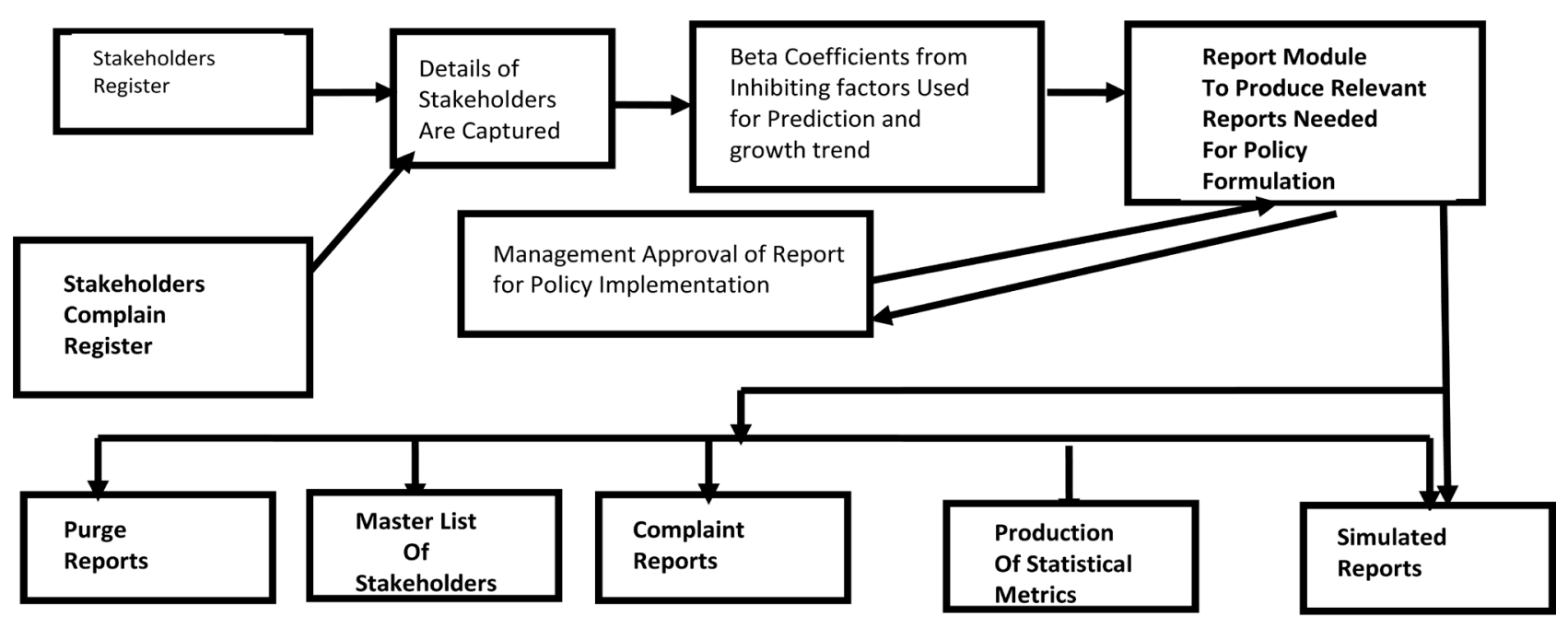

Source: field work 2018.

Figure 1. High level model of the proposed IPV6 predictive model. 
Table 1. Inhibiting factors from $\left(M_{1}-M_{9}\right)$.

BASE GROWTH YEAR: 2017-2027 (10 years). MIGRATION TO IPV6 GROWTH TRAJECTORY SCENARIO: INHIBITING FACTORS FROM $\left(M_{1}-M_{9}\right)$

\begin{tabular}{cccccccccc}
\hline YEAR & $M_{1}$ & $M_{2}$ & $M_{3}$ & $M_{4}$ & $M_{5}$ & $M_{6}$ & $M_{7}$ & $M_{8}$ & $M_{9}$ \\
\hline 2018 & 6 & 5 & 6 & 6 & 6 & 6 & 6 & 6 & 6 \\
2019 & 11 & 11 & 11 & 12 & 12 & 12 & 11 & 12 & 12 \\
2020 & 17 & 16 & 17 & 18 & 28 & 17 & 17 & 18 & 19 \\
2021 & 22 & 21 & 23 & 24 & 24 & 23 & 23 & 23 & 25 \\
2022 & 28 & 27 & 28 & 30 & 30 & 29 & 28 & 29 & 31 \\
2023 & 33 & 32 & 34 & 36 & 36 & 35 & 34 & 35 & 37 \\
2024 & 39 & 37 & 40 & 42 & 42 & 41 & 39 & 41 & 43 \\
2025 & 44 & 43 & 46 & 47 & 48 & 47 & 45 & 47 & 49 \\
2026 & 50 & 48 & 51 & 53 & 54 & 52 & 51 & 53 & 56 \\
2027 & 55 & 54 & 57 & 59 & 60 & 58 & 56 & 58 & 62
\end{tabular}

$M_{1}=$ NO formal education on IPV6 awareness and capacity building. $M_{2}=$ clumsy Resources and Development (R \& D), budget priority and Administrative procedures. $M_{3}=$ Allaying fears on transition cost and non-request from end users. $M_{4}=$ lack of technical knowhow/Expertise in installing IPV6 transition mechanism. $M_{5}=$ Difficulty of security issues fixed-intrusion. $M_{6}=$ lack of IPV6 upstream service providers/Exchange point. $M_{7}=$ lack of core metro and Edge equipment compatibility issues. $M_{8}=$ lack of ISPS' need of a business case in Carrier Grade Router. $M_{9}=$ lack of government support on condition of anonymity-incentive, tax rebate, sponsorship training and on power generation.

Table 2. Inhibiting factors from $\left(M_{1}-M_{9}\right)$.

Migration to IPV6 Growth Trajectory prediction

Base Growth year: 2017 to 2027 (10) Years Trajectory.

Scenario: inhibiting factors from $\left(M_{1}-M_{9}\right)$

\begin{tabular}{cccccccccc}
\hline Year & $M_{1}$ & $M_{2}$ & $M_{3}$ & $M_{4}$ & $M_{5}$ & $M_{6}$ & $M_{7}$ & $M_{8}$ & $M_{9}$ \\
\hline 2018 & 12 & 15 & 12 & 15 & 12 & 15 & 12 & 12 & 12 \\
2019 & 23 & 29 & 24 & 30 & 24 & 30 & 24 & 24 & 25 \\
2020 & 35 & 44 & 35 & 45 & 36 & 45 & 36 & 36 & 37 \\
2021 & 47 & 58 & 47 & 60 & 48 & 60 & 48 & 48 & 49 \\
2022 & 58 & 73 & 59 & 76 & 60 & 75 & 60 & 60 & 62 \\
2023 & 70 & 87 & 71 & 91 & 73 & 90 & 72 & 72 & 74 \\
2024 & 82 & 102 & 83 & 106 & 65 & 105 & 84 & 84 & 86 \\
2025 & 93 & 116 & 94 & 121 & 67 & 120 & 96 & 96 & 98 \\
2026 & 105 & 131 & 106 & 136 & 109 & 135 & 109 & 108 & 111 \\
2027 & 117 & 145 & 118 & 151 & 121 & 150 & 121 & 120 & 123
\end{tabular}

$M_{1}=$ NO formal education on IPV6 awareness and capacity building. $M_{2}=$ clumsy Resources and Development (R \& D), budget priority and Administrative procedures. $M_{3}=$ Allaying fears on transition cost and non-request from end users. $M_{4}=$ lack of technical knowhow/Expertise in installing IPV6 transition mechanism. $M_{5}=$ Difficulty of security issues fixed-intrusion. $M_{6}=$ lack of IPV6 upstream service providers/Exchange point. $M_{7}=$ lack of core metro and Edge equipment compatibility issues. $M_{8}=$ lack of ISPS' need of a business case in Carrier Grade Router. $M_{9}=$ lack of government support on condition of anonymity-incentive, tax rebate, sponsorship training and on power generation.

Scenario 2: Trajectory graph as shown in Figure 2:

Migration to IPV6 Growth Trajectory prediction 
3) SCENARIO III: the test data for scenario III as given below and the results as shown in Table 3; and Figure 3:

$M_{1}=-0.181, M_{2}=-0.175, M_{3}=-0.186, M_{4}=-0.194$,

$M_{5}=-0.196, M_{6}=-0.190, M_{7}=-0.184, M_{8}=-0.191$,

$M_{9}=-0.202:$ Base year $=2017$, year $\mathrm{Gap}=\operatorname{ten}(10)$ years

Table 3 shows when the inhibiting factors get worse or adjusted negatively.

Migration to IPV6 Growth Trajectory prediction

Scenario 3: Trajectory Graph as shown in Figure 3:

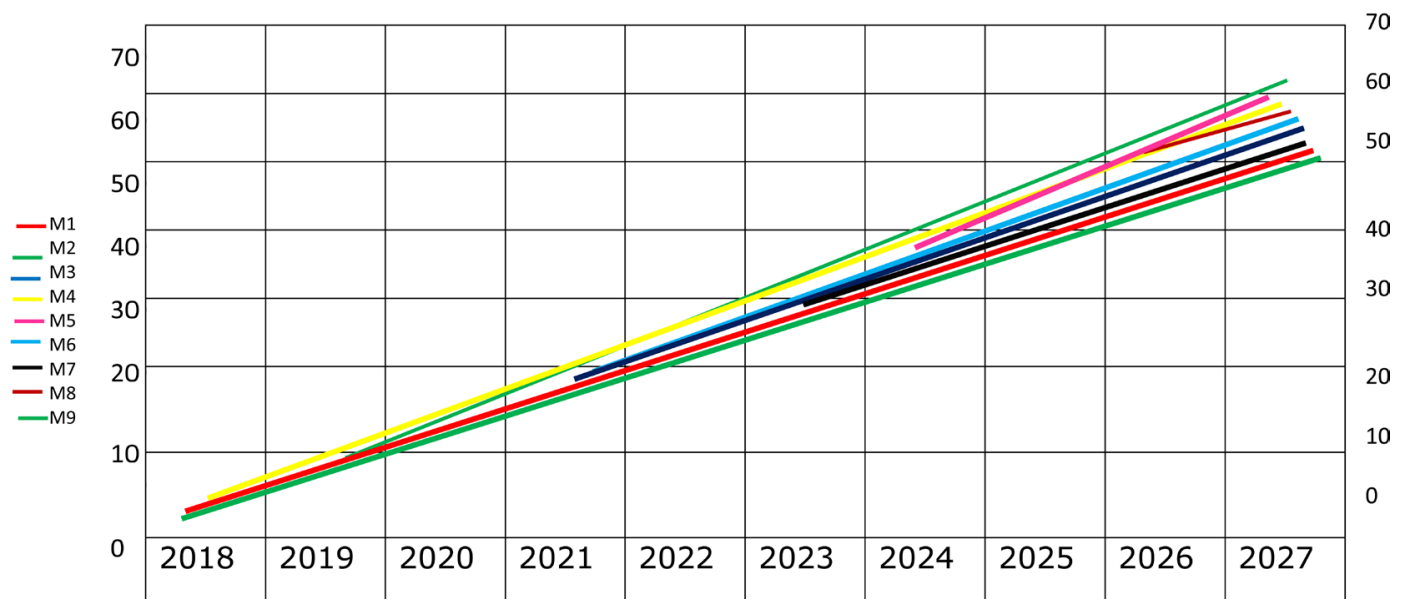

Figure 2. Scenario I data output/graph.

Table 3. Inhibiting factors from $\left(M_{1}-M_{9}\right)$.

Base Growth year: 2017 to 2027 (10) years. Migration to IPV6 growth

Trajectory:

Scenario: inhibiting factors from $\left(M_{1}-M_{9}\right)$

\begin{tabular}{cccccccccc}
\hline Year & $M_{1}$ & $M_{2}$ & $M_{3}$ & $M_{4}$ & $M_{5}$ & $M_{6}$ & $M_{7}$ & $M_{8}$ & $M_{9}$ \\
\hline 2018 & -6 & -5 & -6 & -6 & -6 & -6 & -6 & -6 & -6 \\
2019 & -11 & -11 & -11 & -12 & -12 & -12 & -11 & -12 & -12 \\
2020 & -17 & -16 & -17 & -18 & -18 & -17 & -17 & -18 & -19 \\
2021 & -22 & -21 & -23 & -24 & -24 & -23 & -23 & -23 & -25 \\
2022 & -28 & -27 & -28 & -30 & -30 & -29 & -28 & -29 & -31 \\
2023 & -33 & -32 & -34 & -36 & -36 & -35 & -34 & -35 & -37 \\
2024 & -39 & -37 & -40 & -42 & -42 & -41 & -39 & -41 & -43 \\
2025 & -44 & -43 & -46 & -47 & -48 & -47 & -45 & -47 & -49 \\
2026 & -50 & -48 & -51 & -53 & -54 & -52 & -51 & -53 & -56 \\
2027 & -56 & -54 & -57 & -59 & -60 & -58 & -56 & -56 & -62 \\
\hline
\end{tabular}

$M_{1}=$ NO formal education on IPV6 awareness and capacity building. $M_{2}=$ clumsy Resources and Development (R \& D), budget priority and Administrative procedures. $M_{3}=$ Allaying fears on transition cost and non-request from end users. $M_{4}=$ lack of technical knowhow/Expertise in installing IPV6 transition mechanism. $M_{5}=$ Difficulty of security issues fixed-intrusion. $M_{6}=$ lack of IPV6 upstream service providers/Exchange point. $M_{7}=$ lack of core metro and Edge equipment compatibility issues. $M_{8}=$ lack of ISPS need of a business case in Carrier Grade Router. $M_{9}=$ lack of government support on condition of anonymity-incentive, tax rebate, sponsorship training and on power generation. 


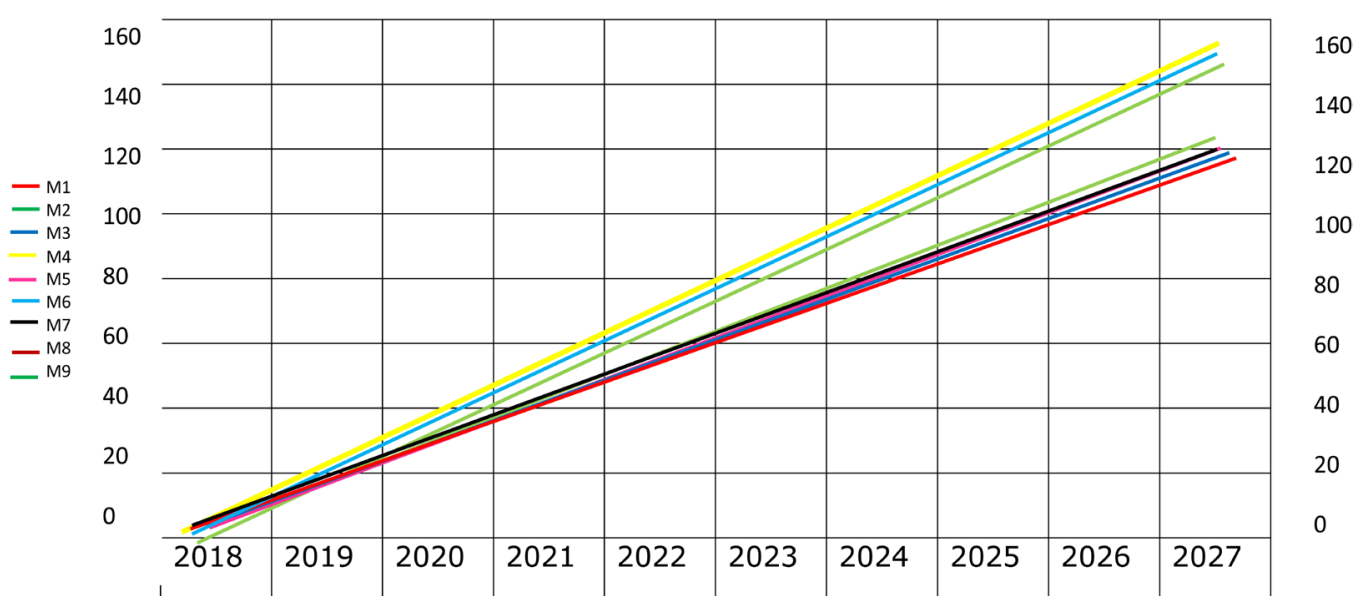

Figure 3. Scenario II data output/graph.

\section{Evaluation Criteria}

The indices derived from the statistical analysis were deployed into our model: $Y=a+B_{1} x_{1}+B_{2} x_{2}+\cdots+B_{n} x_{n}$ (8.1) above, translated and finally evaluated using the model: $Y=a+\beta_{m 1} x_{1}+\beta_{m 2} x_{2}+\cdots+\beta_{m n} x_{n}$ (adjusted equation) with input Beta values of $\left(M_{1}-M_{9}\right)$, resulting to the production into the IPV6 predictive model (IPV6 pm) which is based on multiple linear regression model.

where: $Y=$ migration to IPV6 = the dependent variable, (value for the year being predicted); $a=$ constant; $M_{1}-M_{n}$ are the government policies on (inhibiting factors); and $X_{1}-X_{n}=$ independent variables (the period of forecast), and $\beta$ is the Beta coefficients which measures growth index. And this is applied in the three different scenarios;

1) As it is now with government policies on (inhibiting factors) on migration to IPV6.

2) Hypothetical adjustments of the Beta indices if government policies on (inhibiting factors) are removed or improved upon.

3) Hypothetical adjustments if government policies on (inhibiting factors) becomes stiffer or gets worse.

The three Scenarios demonstrated expected growth or decline of migration to IPV6.

\section{Discussion of Results}

Comparing the results of the above three Scenarios: In scenario one, the results of migration to IPV6 Non motivation in Nigeria has a relationship with the government policies (inhibiting factors) like: No formal education on IPV6 awareness, capacity building and on the benefits of IPV6 $\left[M_{1}\right]$; Clumsy Resources and Development ( $\mathrm{R} \& \mathrm{D})$, budget priority and administrative procedures $\left[M_{2}\right]$, Allaying fears on transition cost and non-request from end users. $\left[M_{3}\right]$, Lack of technical knowhow/Expertise in installing IPV6 transition mechanism $\left[M_{4}\right]$, Difficulty of security issues fixed-intrusion $\left[M_{5}\right]$, Lack of IPV6 upstream service providers/Exchange point $\left[M_{6}\right]$, Lack of core-metro and Edge equipment compatibility issue $\left[M_{7}\right]$, Lack of ISPs' need of a business case-carrier Grade Router 


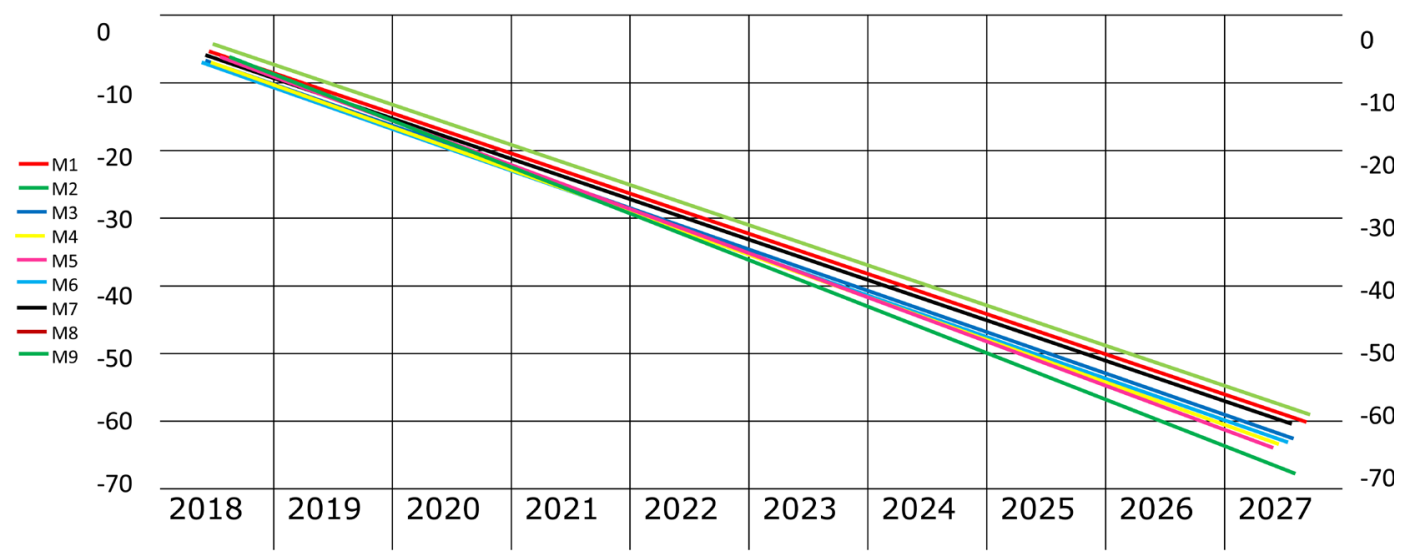

Figure 4. Scenario III data output/graph.

$\left[M_{8}\right]$, Lack of government support on the condition of anonymity-incentives, tax rebates, sponsorship training and on power generation $\left[M_{9}\right]$. And other policies as identified during systems analysis, justifies that inhibiting factors are responsible for apathy to migration to IPV6 in Nigeria. See Figure 2 of scenario one above and as shown in Figure 2, sample output. Adjusting the indices in scenario one positively (scenario two) indicating the removal or improvement on the above policies (inhibiting factors) shows that migration to IPV6 improved and may become feasible and viable. And this is as expected. The result is as shown in Figure 3 of scenario two above, sample output. In scenario three, when the indices were adjusted negatively to test the future of migration to IPV6 if government policies (inhibiting factors) worsened, the result also indicates that if government still stiffens their policies (inhibiting factors) that migration to IPV6 will collapse completely. This is as indicated by Figure 4 of scenario three above and as shown in Figure 4, sample output. Finally, the results of the in-depth analysis and further synthesis of the actual result and the expected result described herein, provided a further insight and clear indication that the test data was true reflection of the challenges of migration to IPV6 in Nigeria and thus that the software performed as expected.

\section{Conclusions}

As challenging as this research problem is, efforts were made to assess the feasibility and viability of identifying factors that can encourage migration to IPV6 and use the factors to forecast growth. This serves as a tool for policy formulation in Nigeria. Real-time interviews were conducted to capture real life feedback from stakeholders to strengthen the quality of analysis and close any gap in predictive model. In fact, a hypothetic deductive method was employed to collect field data from stakeholders and these data were analyzed using SPSS analysis.

A model for predicting migration from IPV4 to IPV6 is undoubtedly the concept that can bring about the desired IPV6 growth in the Nigerian's telecommunication market, not only because it can provide improvement in the web transaction but also, as uncovered in this study. It will proliferate creativity among 
service stakeholders to embrace migration to IPV6, and value added services to remain competitive. As Nigeria is playing catch-up in the evolution of the migration from IPV4 to IPV6 scheme already successful in many countries, the government has a strategic role to play. Government's role should focus on providing strategic directives using functional by-laws that will provide enlightenment and trust by the members of the public. The research conducted in this study has indicated that stakeholders are more privy to embrace the migration to IPV6 scheme if government ameliorates the bottlenecks in the migration process to IPV6. But if government allows the existing inhibiting factors on migration process to IPV6 adoption to remain, stakeholders may not be motivated to embrace migration to IPV6. The reduction or elimination of most of the inhibiting factors makes migration to IPV6 feasible and viable. And of course, should government further stiffen the current inhibiting factors, migration to IPV6 will totally die in the not distant future.

\section{Recommendations}

1) The government should plan to develop the regulatory, legal and technical structure for the migration and implementation of IPV6 scheme in Nigeria and also establish commissions (body), education and research institutions, steering committee, and project task team, as well as creating awareness of the emergence of migration to IPV6, benefits and capacity building In Nigeria.

2) Government needs to appoint this steering committee and a project team based on experience, qualification, Expertise and must be devoid of any political agenda, who will help government to create new laws and legislation that will afford internet stakeholders and its service provisions with legal certification.

3) This committee and the team will also carry out migration to IPV6 processes and redesign that, which will provide government, the stakeholders and public good deliverables.

4) A law should be enacted in line with certain provisions of certain sections of the selected body's Act Number, which vests the body with the exclusive right to regulate the migration process to IPV6 in Nigeria.

5) The monitoring and the enforcement of compliance with regulations in order to ensure fair and equity in the migration process and competitions must be considered.

6) The frame work will spell out the inhibiting factors that are responsible for apathy to migration to IPV6 and decides optimal solutions, and also the frame work will spell out the migration process rules for implementing the government objectives of protecting the stakeholders' interest in migration process.

7) Government should support the stakeholders on the condition of anonymity-incentives, tax rebates, sponsorship training and on power generation. This will be a Revolutionary step in towards migration to IPV6 in Nigeria.

\section{References}

[1] ARIN Board of Trustee (2007) Migrations to IPV6 Impending Internet Address 
Shortage; a Resolution on Internet Technical Community. America Registry for Internet.

[2] Olawale Ige (2011) Guardian Wednesday, June 8, 2011. P.25.

[3] Okafor, E.C. (2001) Starting with Computer. Immaculate Publication LTD. Enugu.

[4] Osuagwu, O.E (2009) Insight into the New Frontiers of Computer Forensics, Cyber-Criminality and Internets, Oliverson Industrial Publishing House (OIPH), 9 Mbonu Ojike Street, Owerri-Nigeria.

[5] Wong and Anada (2002) Internet Protocol Version 6, SINGAREN Production, Singapore.

[6] Susan Craw Ford (2007) Biggest Threat to Internet and Massive Virtual Blackout, an Outspoken Board Member Internet Corporation of Names and Numbers, Hudson Institute.

[7] Federal Agencies (2007) IPV6, Don't Get Left on the Platform; an Advocate For Web Upgrade.

[8] Field, A. (2005) Discovering Statistics Using SPSS. Sage, London. 